\title{
An asynchronous approach to teaching Math and Engineering software within the context of a course in Mechanisms
}

\begin{abstract}
In this work we describe the use of interactive, web-based instructional technologies in conjunction with in a course in Mechanisms to teach math and engineering software, and vice-versa, the use of interactive, web-based instructional technologies in conjunction with math and engineering software to teach in a course in Mechanisms. The idea is to accomplish both the teaching of the course content and the use of math and engineering software without taking class time to teach the software. The guiding principle is to initially introduce the student one step at a time through only the parts of the software necessary to solve the specific problem at hand, be it a lecture concept/learning objective or a homework assignment. Then, after the student has developed a certain familiarity with the software, they can more readily use the software's own more encyclopedic assistive materials to address new and more comprehensive tasks. The approach taken incorporates the use of "screen capture with audio" avi tutorials, along with a variety of interactive materials including; supplemental lecture notes, homework assignments and solutions, sample exams and exam solutions, and projects. Liberal use is also made of the software packages' own assistive materials. The software "taught" includes MathSoft's Mathcad and MSC's Working Model 2D, with TechSmith's Camtasia used to create the "screen capture with audio" avi files.

Examples demonstrating the delivery and instructional techniques used are given. The first two examples illustrate the interactive lecture and homework materials. The final example shows part of an interactive sample project involving the synthesis/design and subsequent analysis of a planar four bar linkage.

\section{Introduction}

The general demand for fewer hours in the curriculum without sacrificing content, along with the desire for the development of a working knowledge of math and engineering software, requires "new" delivery approaches. The development of a web-site with various modes/types of asynchronous tutorial material is the approach taken in this course. More specifically, Word documents with hyperlinks to "screen capture with audio" avi tutorials, and interactive Mathcad and Working Model 2D (WM2D) example "scripts" are used as the delivery modes. TechSmith's Camtasia is used to create the avi tutorials. This form of presentation is very effective for teaching GUI type software such as WM2D. While not as effective for Mathcad, it is still beneficial to the student to have a verbal dialog along with a "textual" presentation of how to access and use certain features of the software. Beyond the development of such asynchronous materials is the "Bottom Line": The students must want to use the materials. Our perspective is to first hook them with the utility of the software to solve problems and understand concepts through in-class demonstrations, then teach them the software in easily digestible bites using asynchronous tutorial materials that coincide with course assignments.
\end{abstract}

An outline of the course content followed by examples demonstrating the delivery and instructional techniques used are given. The first two examples illustrate the interactive lecture and homework materials. The final example shows part of an interactive sample project involving the kinematic synthesis and subsequent analysis of a planar four bar linkage. In order to more easily discriminate 
between the textual content of this paper and the asynchronous materials, the textual content is shown in red.

\section{MECE 3380 - Kinematics and Dynamics of Machines}

This course is aimed at introducing Junior-level students to issues involved in the kinematic synthesis, dynamic analysis, and "optimal" design of co-planar mechanisms, with an emphasis on four bar linkages. The course content is summarized in Table 1.

Introduction

\section{KINEMATICS AND DYNAMICS OF MACHINES}

Fundamental Concepts; Joint \& Link Types, DOF

Linkage Classification, DOF \& K. Equiv. Diagrams

1-DOF Mechanism Mode1 ( $\mathrm{KIC}$-based)

Survey of Simple Mech. Devices;

- Constant Gain;

- Constant but Adjustable Gain.

- Cams, Differentials \& Misc.

- Epicyclics

Design Theory and Methodology and Gear Train Examples

Dimensional Synthesis; Concepts

Tools; Curvature Transform

- Coplanar Motion Synthesis

- Coplanar Motion Synthesis

Solvable Tasks;

- Cognates - Angular \& Path

- 2 Link Chain, 2 Cranks, \& Slider and Crank

- 2 Cranks via Inversion

- Path Coordination with a Crank

- Other Synthesis Techniques

4R-4Bar Analysis

- Position Analysis

- Velocity., Acceleration., and Force Analysis

- Shaking Force and Moment Balancing

Kine matic Influence Coefficient (KIC)-Based Dynamic Modeling

- Mass and Externally Applied Loads

- Springs and Dashpots (Viscous Friction)

- The Controlling Equation \& Transfer of Input

Dyad Based Mechanism Analysis

- Structures

- Position Analysis

- Velocity/G-function \& Acceleration/H-function Anal.

- Force Analysis

Lead in to Machine Elements \& Senior Design

Table 1. Course outline

\section{Lecture Summaries}

Condensed summaries of each lecture are posted on the course web-site with links to additional asynchronous tutorial material as appropriate. A portion of the summary of one lecture is given in Figure 1. In this case the link is to an interactive WM2D simulation, shown in Figure 2, which clearly illustrates the effect that fixing different links in a 4-Bar linkage has on the absolute motion 
of the device. The student is directed to move the anchor to fix the different links, and to simulate the four cases on their own. This provides a very simple introduction to interacting with WM2D. In general, links exist to textual material, Camtasia tutorials, Mathcad, and/or WM2D.

II. Gxashof Criteria and Range of Mo tion of the $4 R-4 B$ ar (More to ceme): This criteria is used to deternine if a 4R-4Bat is a "Crank-Rocker". "Double-Crank", or "Double-Rocker" Groshofs law states thet "the sum of the shortest httpil/wwwiengr.panam,edu/vrafree/ $4 \mathrm{R}-4 \mathrm{Bar}$ caninot be greater than the sum of the lengths of the olloer two links (P \& K\&D/Mech Recog/Grascit1 wmad le rotation between two linke."

\begin{tabular}{|l|}
\hline $5(L+S[m]$ \\
\hline$L+S 4.900$ \\
\hline
\end{tabular}

\begin{tabular}{l} 
Fy $P+Q(\mathrm{~m})]$ \\
\hline+05.000 \\
\hline
\end{tabular}

Grashol Type 1: $L+S<P+Q$

Grark. Piocker. Place anchs on L ou P is is crank]

Drag Link. / Double Ciard: Place anchot on S (S is ground]

Doukle Rioder: Place archot on Q A.irik opposite S is Groundl

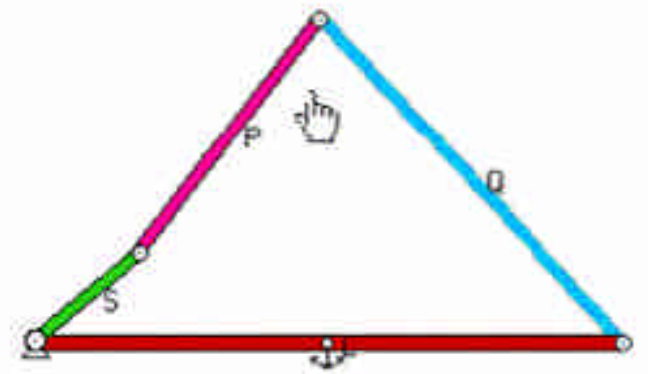

a. Grashof Type I: Continuous relative rotation; $\mathrm{L}+\mathrm{S}<\mathrm{P}+\mathrm{Q}$

i. Crank-Rocker: Two posebilitioe. The crank: is $\mathrm{S}$, with ground being enther of the adjacent links.

ii. Double-Crank: One possibility. $S$ is ground

iii. Double-Racker: One possibility. The ground link is opposite $S$.

Figure 1. Lecture Summary

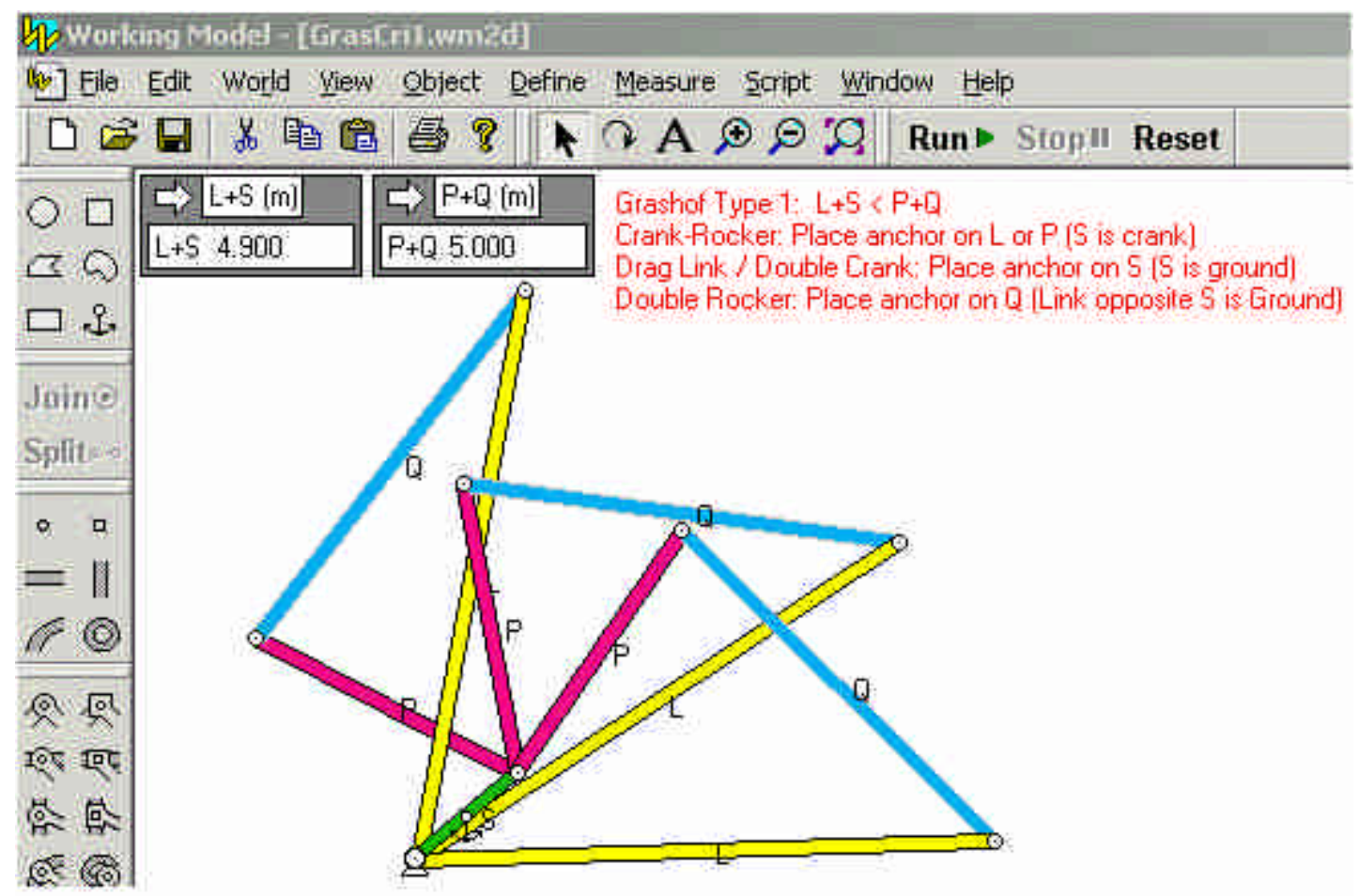

Figure 2. Working Model Simulation of Lecture Concept 


\section{Homework Assignment}

Part of the first homework assignment as posted on the class web site is shown in Figure 3. The use of WM2D is again particularly effective for this assignment dealing mechanism recognition. The link to and use of the Working Model simulation clearly illustrates the motion of the device and thereby the various links and joints that comprise the mechanism. This greatly facilitates the students ability to complete the assignment. Figure 4 shows the solution as posted on the web, the link to the WM2D file is of course still present. Many of the example mechanisms used in this assignment, such as the one selected for illustration and shown in Figures 3 and 4, are taken from Myszka, David H., Machines \& Mechanisms: Applied Kinematic Analysis, $2^{\text {nd }}$ ed., Prentice Hall, 2002.

\section{Homework \#1}

K\&D of Machines

Fall 2002

As described:

I All mechanisms fall into one of three categories:

A. Motion Generation; Rigid-body guidance (position and orientation)

B. Path Generation, Guidance of the path of a point

C. Function Generation, "Control" of the motion (position and/or orientation) or force/torque of one link/body (the output) as a function of the motion or force/torque of another linkbody (the input)

II Some "simple" 1-DOF mechanisms have been given "names", including the 4R-4Bar, the Slider-Crank, the Inverted S-C. Watt 1 and 2, and Stephenson 1, 2, and 3.

III. The general Gruebler equation for determination of the number of degrees-of-freedom (DOF) of a mechanism is $F=3(n-1)-2 j-h$. Recall that there are special cases, including prismatic-loops, passive freedoms, and redundant constraints.

For each of the devices/machines/mechanisms shown below, determine
a. Its "category"
b. Its name (if any)
c. Its DOF

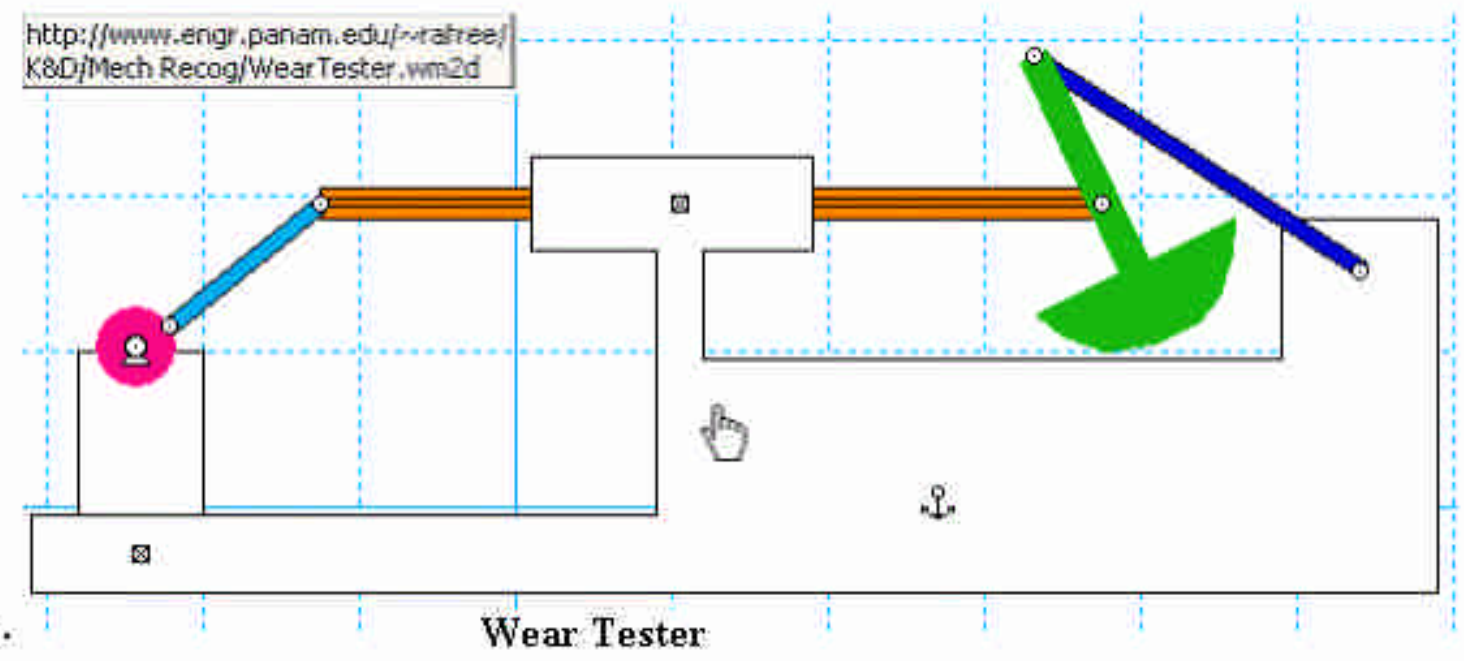

Figure 3. Homework \#1 as posted on the Web-site with Link to WM2D Simulation 


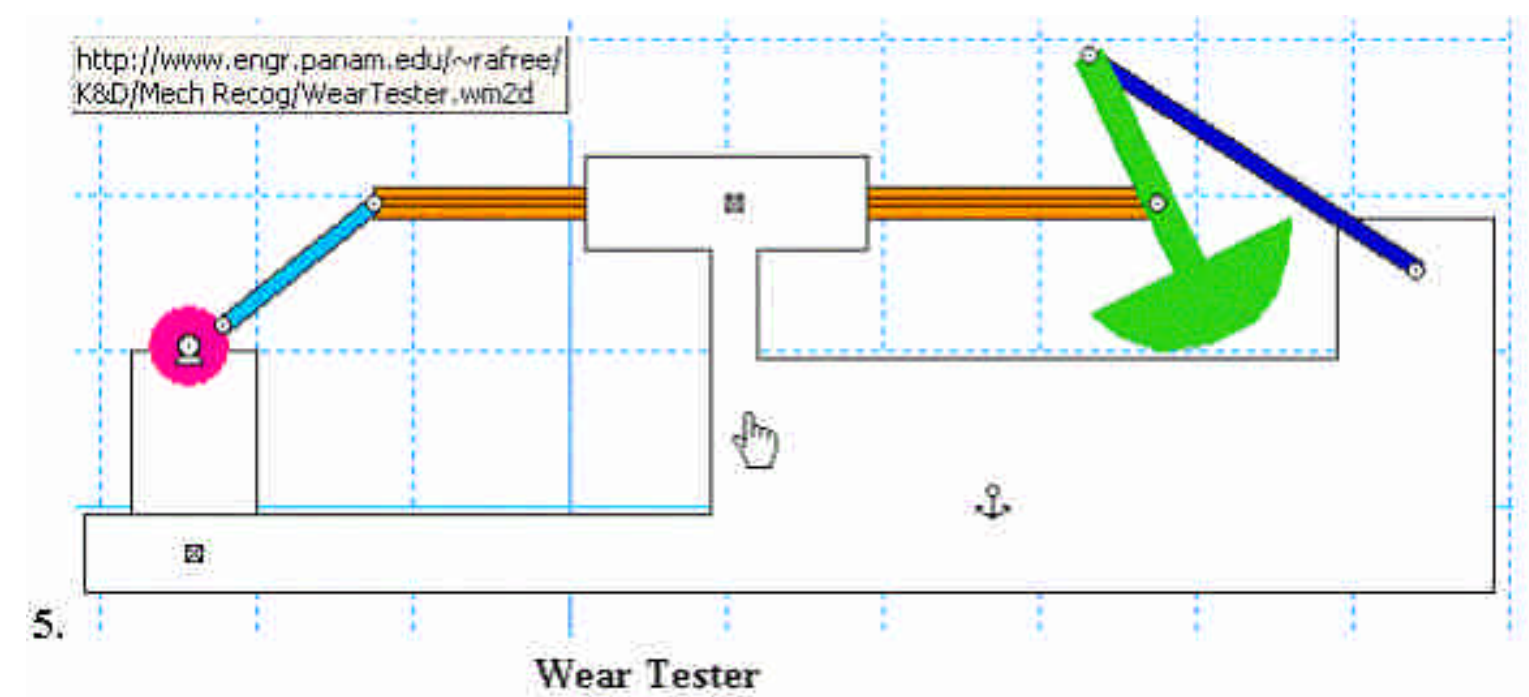

a. The task here is to control the Motion (both position and orientation) of the green "wear link".

b. This is a Watt 2. Ground and the orange link are ternaries and are directly connected through a prismatic joint

c. $\mathrm{n}=6, \mathrm{j}=7, \mathrm{~h}=0, \Rightarrow \mathrm{F}=3(6-1)-2(7)-1(0)=1$

Figure 4. Posted Solution to Home work \#1 with Iink to WM2D Simulation

\section{Project Requirements}

The project requires student teams (limited to 3 students) to propose a task that can be solved using a four revolute 4-Bar (4R-4Bar) linkage. They are encouraged to select a task that can be formulated in terms of either Co-Planar Motion Synthesis, Angular Coordination of Two Cranks, or Path Coordination with a Crank as these procedures are directly treated in class and result in 4R4Bar solution mechanisms. They are then required to obtain two different kinematic solutions to their problem. This is to be done using Mathcad to obtain analytic solutions, and then Working Model 2D to "virtually" verify the solutions. They must also build a physical model of one of the solution 4Bars. After obtaining two valid kinematic solutions they then proceed to perform a dynamic analysis on one of the solutions in one position (state) by hand and with Mathcad. They then use WM2D to dynamically simulate the mechanism. They are required to obtain the same values for the link accelerations, joint reaction forces, the required motor torque that they obtained by hand and with Mathcad. This is intended to verify that they are using WM2D correctly. Finally, they compare their two kinematic solutions dynamically using WM2D under realistic operating conditions for their task and select one as their "optimal" design based on some dynamic metric, such as minimum maximum required input torque magnitude.

\section{Example Project}

The example project discusses a solution to the general task of controlling the motion of the top of a tackle box. In the following, a general overview of the developed tutorial materials is given first, followed by a complete summary of the individual steps required to solve the problem. For brevity, only a few of the associated tutorial materials are graphically illustrated. The synthesis component of the project is presented first, followed by presentation of the analysis component. 


\section{Synthesis}

First, Working Model 2D is used to illustrate a graphical approach to the solution of the "Three Finitely Separated Position" (3FSP) Co-Planar Motion Synthesis (CMS) problem with moving pivot specification. In the process, Camtasia tutorials show the student how to use WM2D to create a 4R-4Bar. Second, a Mathcad script is used to analytically solve the same 3FSP problem. The student is shown (by textual descriptions within the script and with the aid of the Mathcad Resource Center) how to reproduce the script, and how to use Mathcad to solve systems of linear and nonlinear equations, and to create 3D scatter plots with line generation.

The student starts by linking to the tutorial materials from the solution 4Bar shown in Figure 5 and proceeding in a sequential manner through the tutorials listed below.

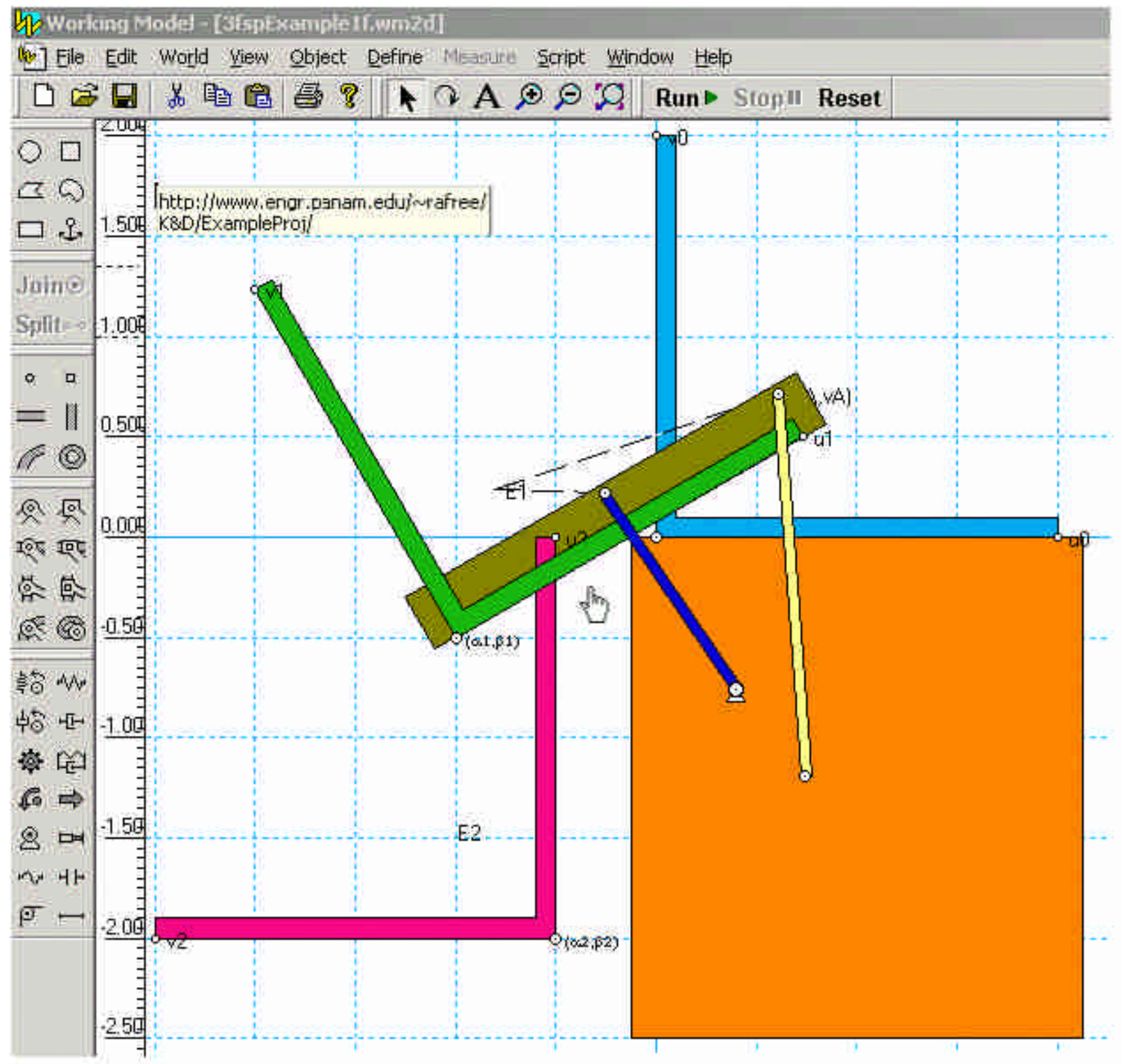

Figure 5. WM2D Simulation of Sample Project Solution with Link to Tutorial Materials File 


\section{Solution Steps / Tutorials List:}

1. Three finitely separated positions (3FSP) of the box top (actually a frame attached to the box top) are specified and shown in WM2D relative to the box.

2. A Camtasia tutorial aimed at showing how to create and position the frames is then viewed.

3. Two points A and B fixed to the box top (the moving pivots of the resulting 4-Bar) are then arbitrarily selected/specified.

4. A Camtasia tutorial, shown in Figure 6 explains how Working Model assigns body-fixed reference frames, and how to locate body-fixed pin joints relative to that frame.

5. A series of figures generated using WM2D and ending with Figure 7, illustrating a graphical procedure for solving the 3FSP CMS problem is then given.

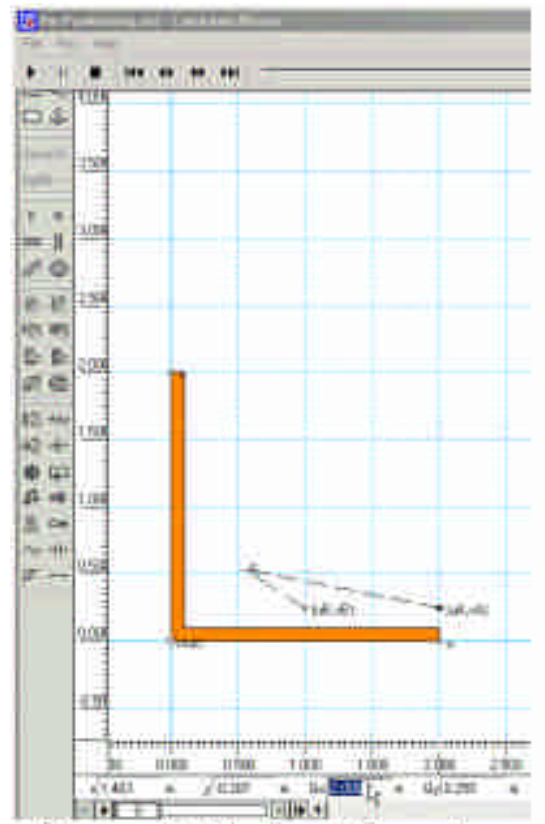

Figure 6. Pin Joint Location

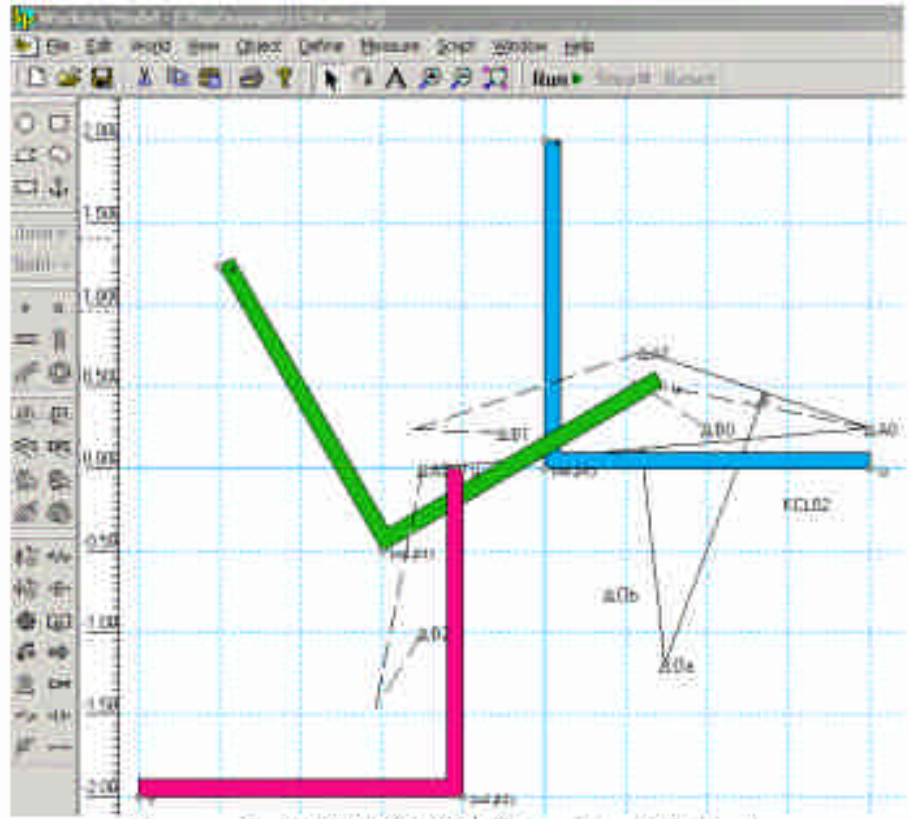

Figure 7. 3FSP-CMS Graphical Solution

6. The resulting 4R-4Bar is then shown in one of the specified positions, as seen in Figure 8.

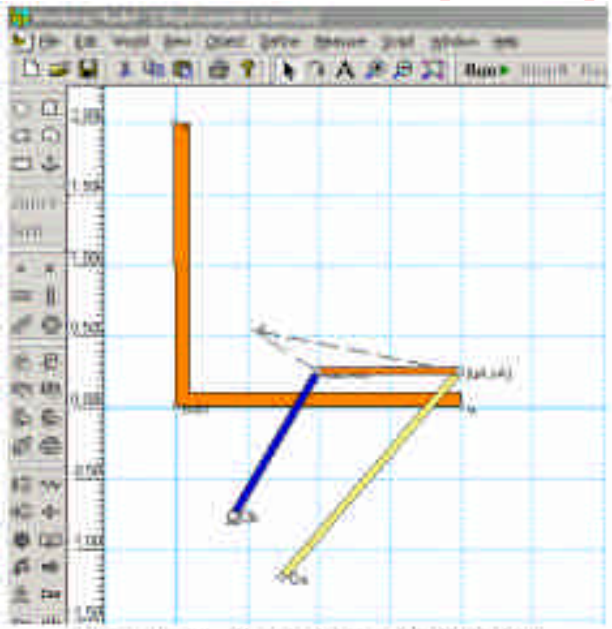

Figure 8 Resulting $4 \mathrm{R}-4 \mathrm{~B}$ ar 
7. Next, a WM2D simulation showing that the 4R-4Bar does indeed take the box top through the three specified positions is run, as illustrated in Figure 9.

8. Finally, with regard to WM2D, the student is to view a Camtasia tutorial, Figure 10, showing the construction of a complete 4R-4Bar, including how to obtain measures of various parameters and to how to control a rotational motor.
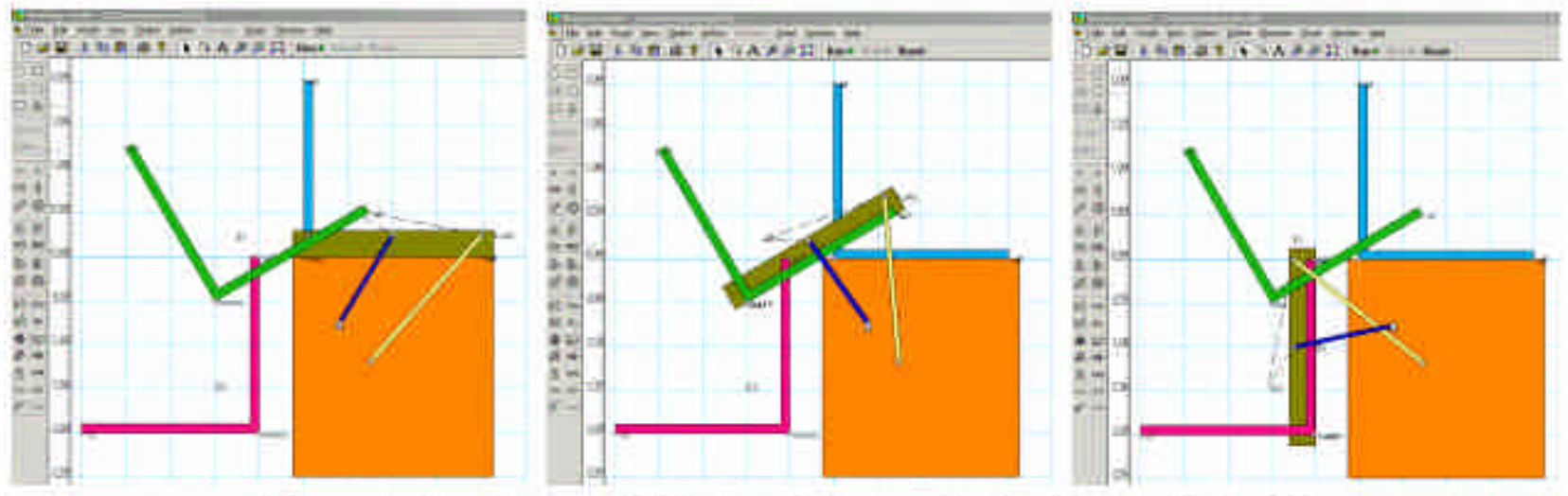

Figure 9. Simulation of Solution 4Bar attached to Box and Box Top:

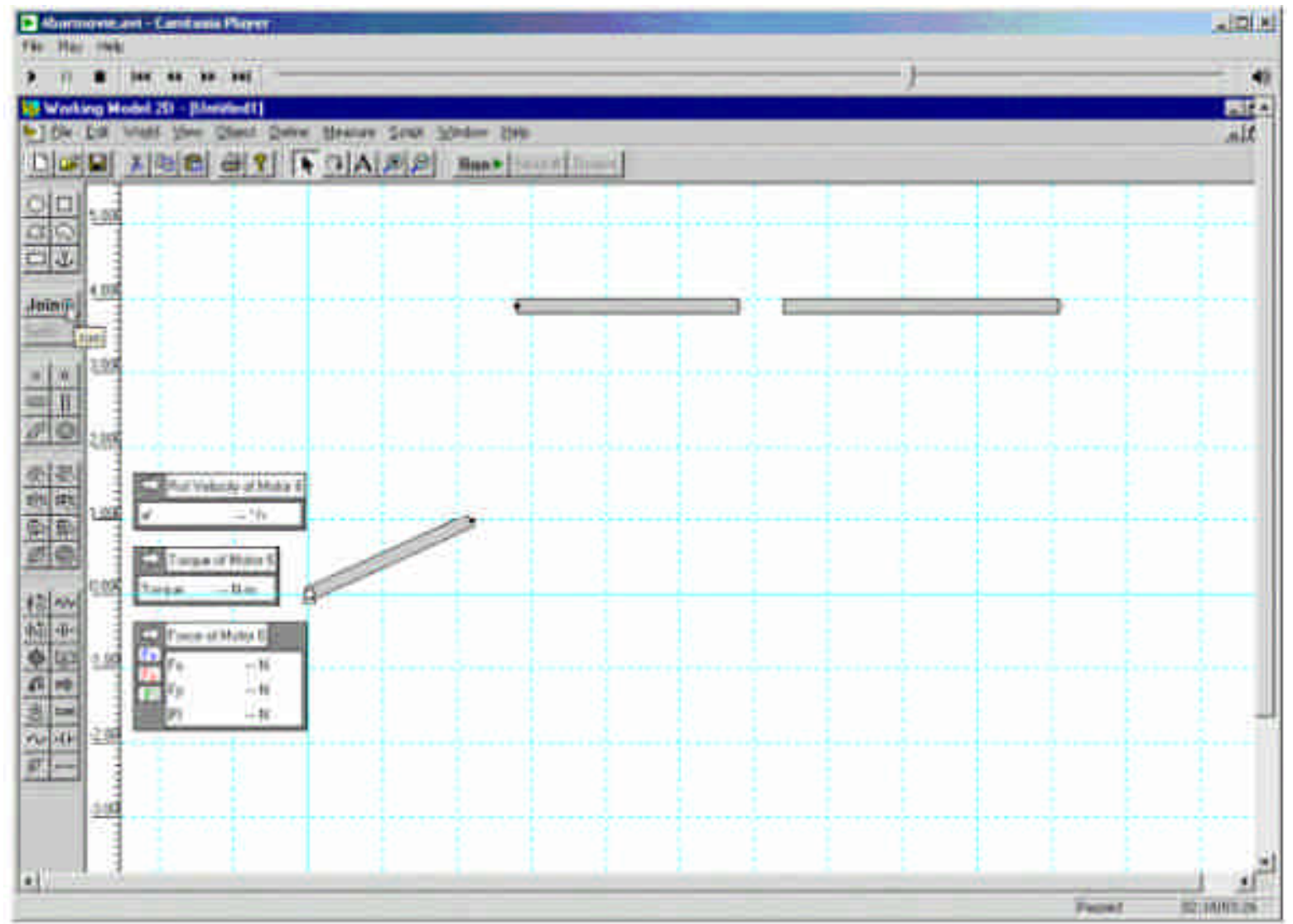

Figure 10, Camtasia Tutorial of $4 \mathrm{R}-4$ Bar Construction 
9. The student now proceeds to view a Camtasia tutorial describing a Mathcad script used to analytically solve the same 3FSP-CMS problem that was just solved graphically. In the process, the students are "shown" the details of how to reproduce the script . For example, there are direct textual directions, as well as, directions as to how to use Mathcad's Resource Center given in the script to assist in this process as illustrated in Figures 11 and 12.

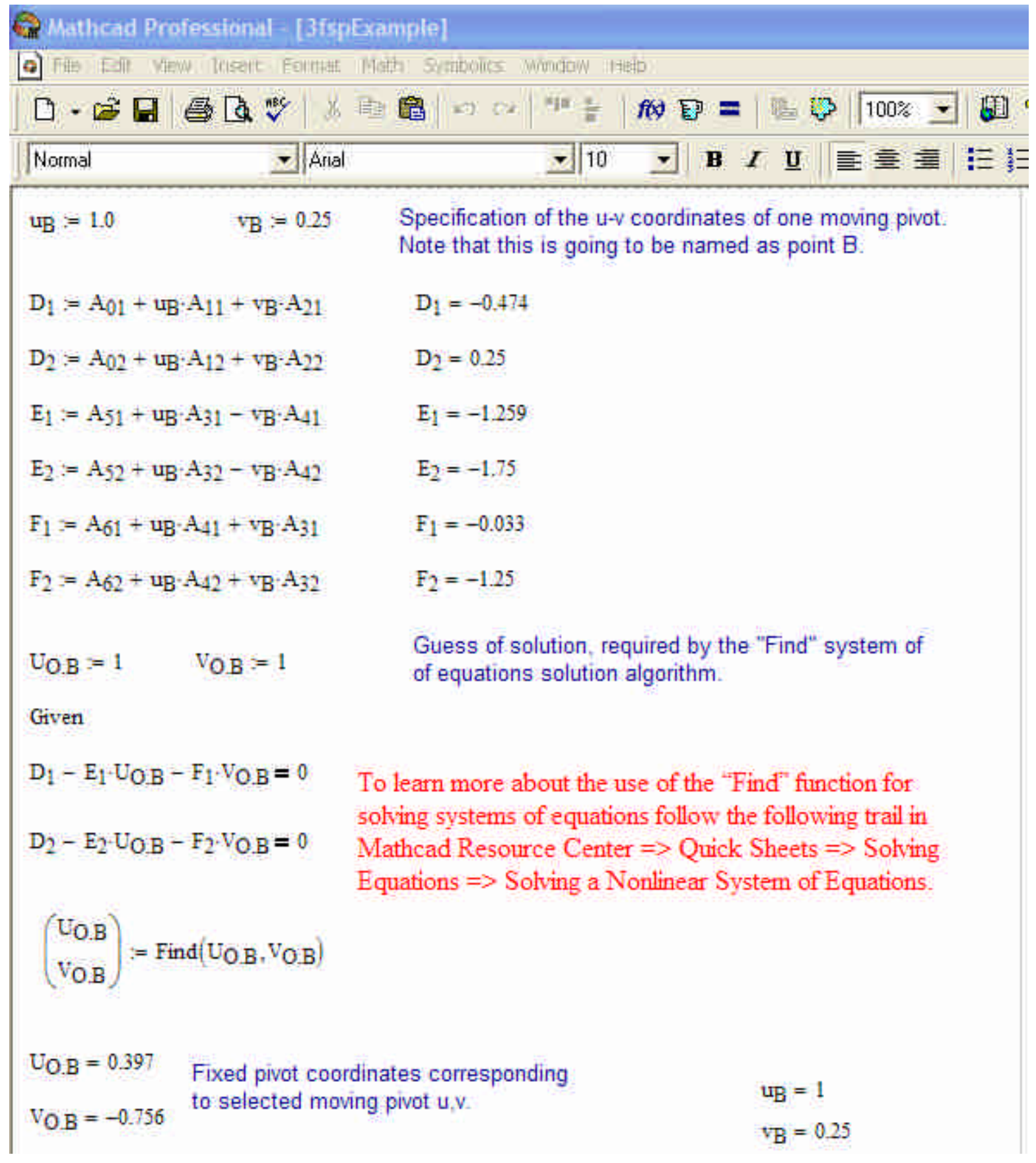

Figure 11. Mathcad script with Directions to the use of Mathcad's Resource Center 

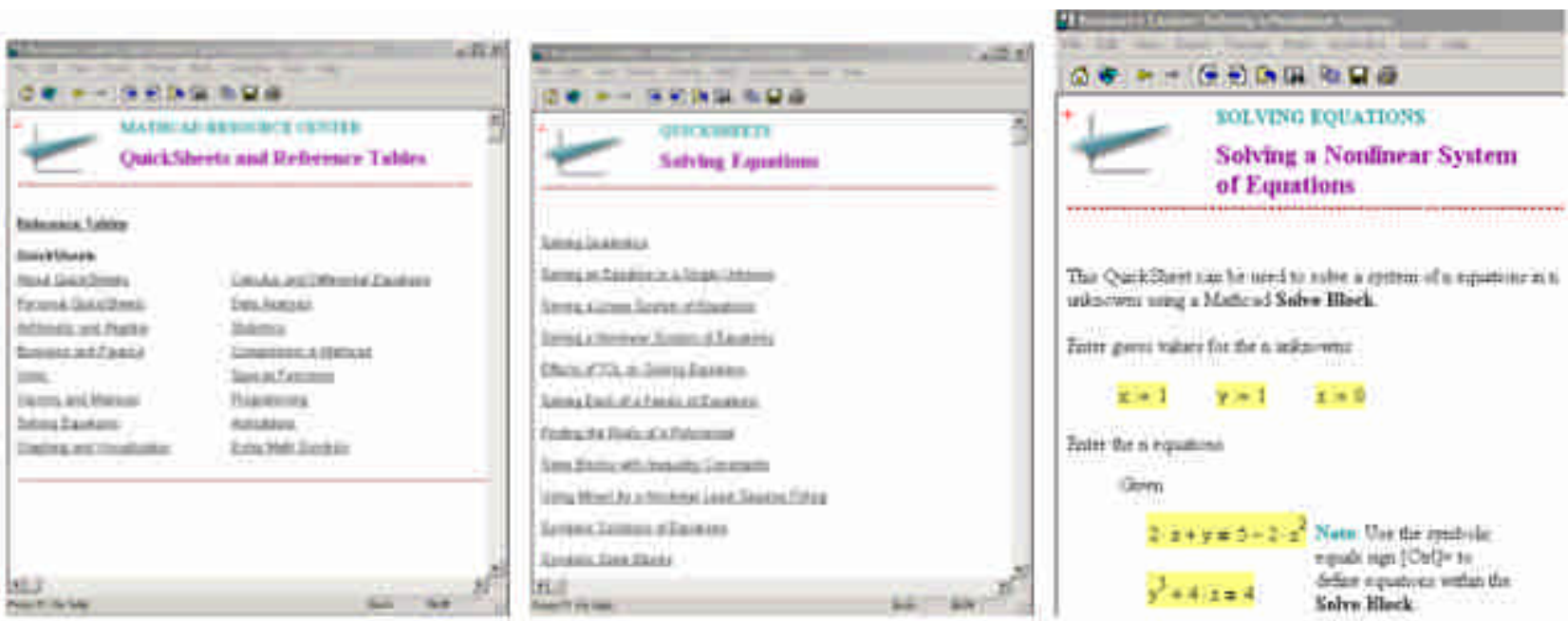

Figure 12. Mathcad Resource Center for use of "Find" function

10. Finally, with respect to Mathcad, to display the 4Bar solution in Mathcad, a description of how to use the 3D Scatter Plot is given. This is illustrated in Figures 13 and 14.

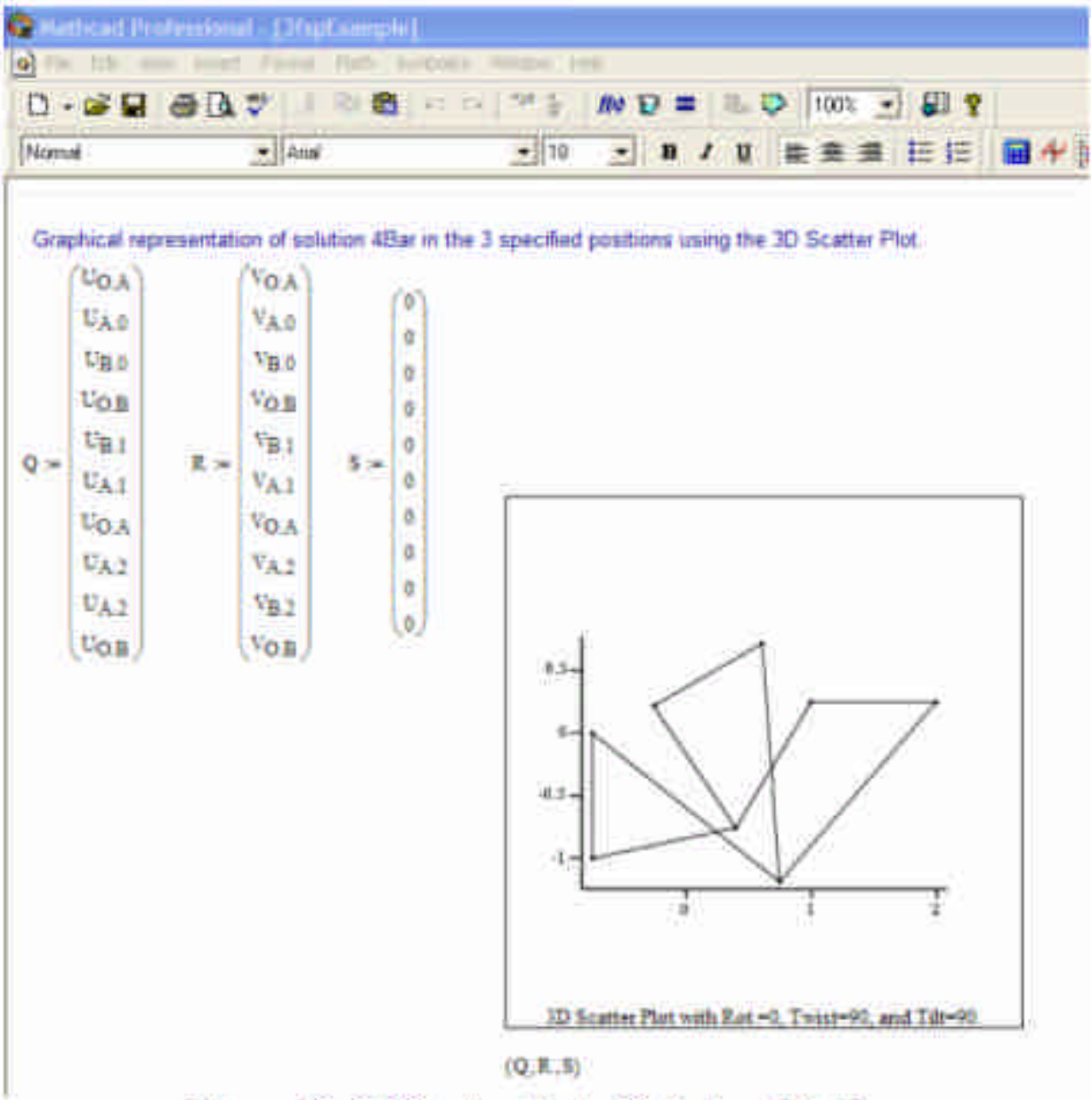

Figure 13. 3D Scatter Plot of Solution 4R-4Bar 


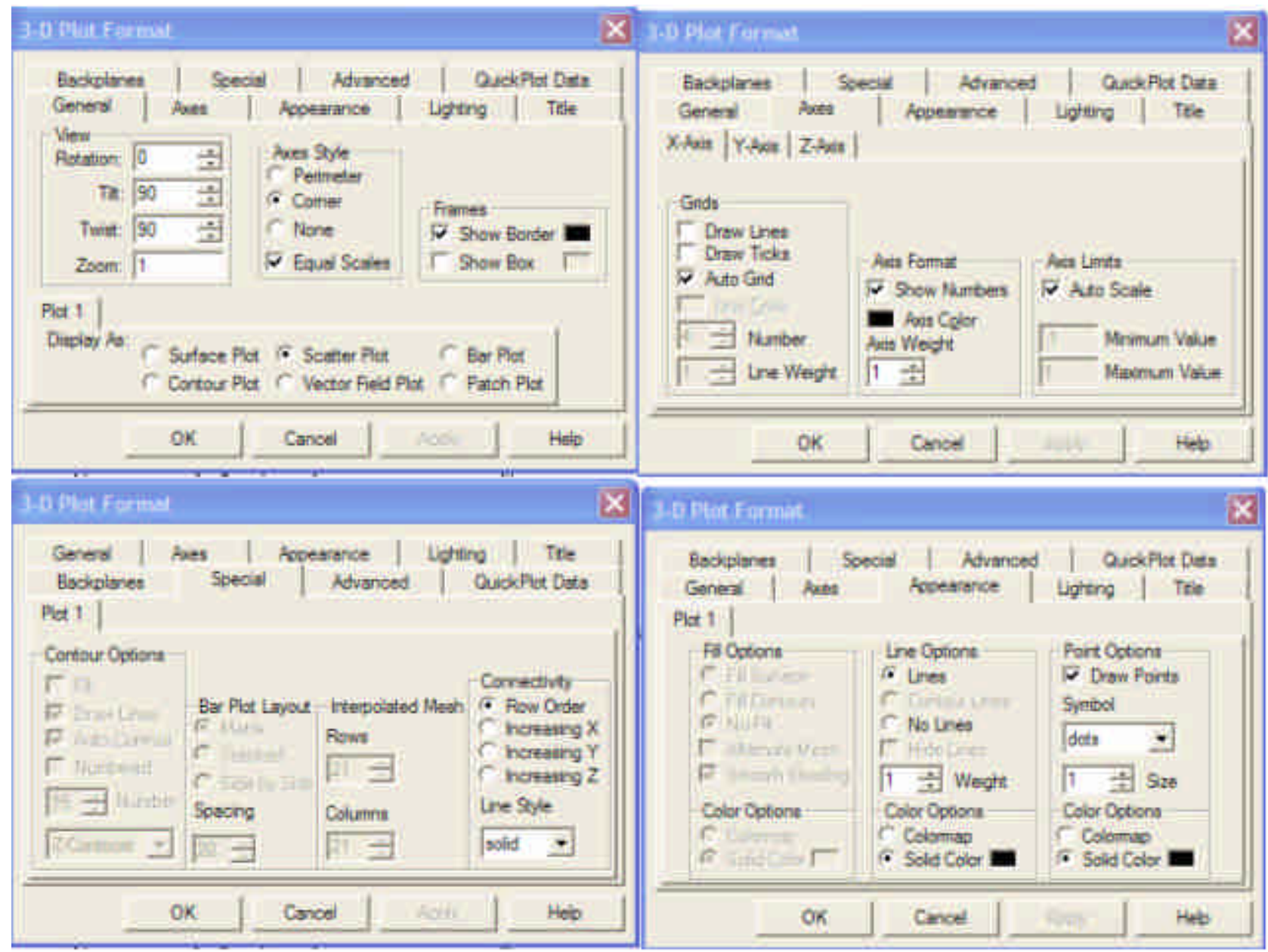

Figure 14. Graphical description of 3D Scatter Plot settings

11. Finally, with respect to synthesis, the students view a final Camtasia "video" giving a general overview of the entire synthesis process, including references to the key software components utilized.

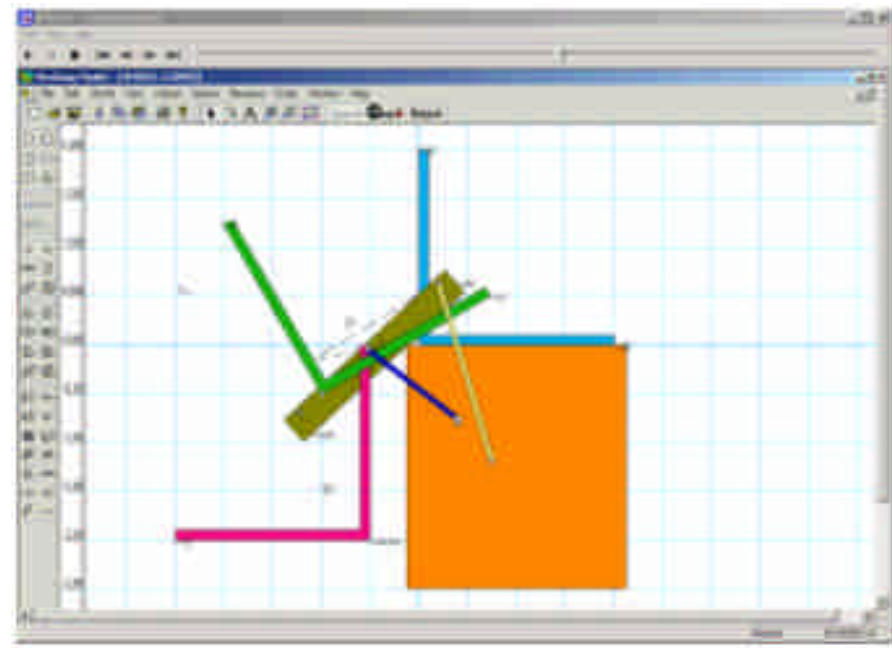

Figure 15. Camtasia Overview of Synthesis Process 


\section{Analysis}

In their project the students are required to create a dynamic simulation of one of their kinematic solutions in WM2D and compare the resulting accelerations, forces, and required motor torque with a corresponding solution obtained using Mathcad. In the process they are shown how to assign masses, inertias, centers of mass, initial velocities, and motor type (acceleration) using Camtasia tutorials. They are also shown how to relate the link frames assigned by WM2D to those used in the development of their Mathcad solution in order to have the centers-of-mass in the same physical locations. These steps / tutorials are again listed below with selected steps illustrated graphically.

\section{Solution Steps / Tutorials List:}

1. The first step is to verify that the initial position of their WM2D simulation corresponds to the position solution obtained using their Mathcad script as illustrated in Figure 16.

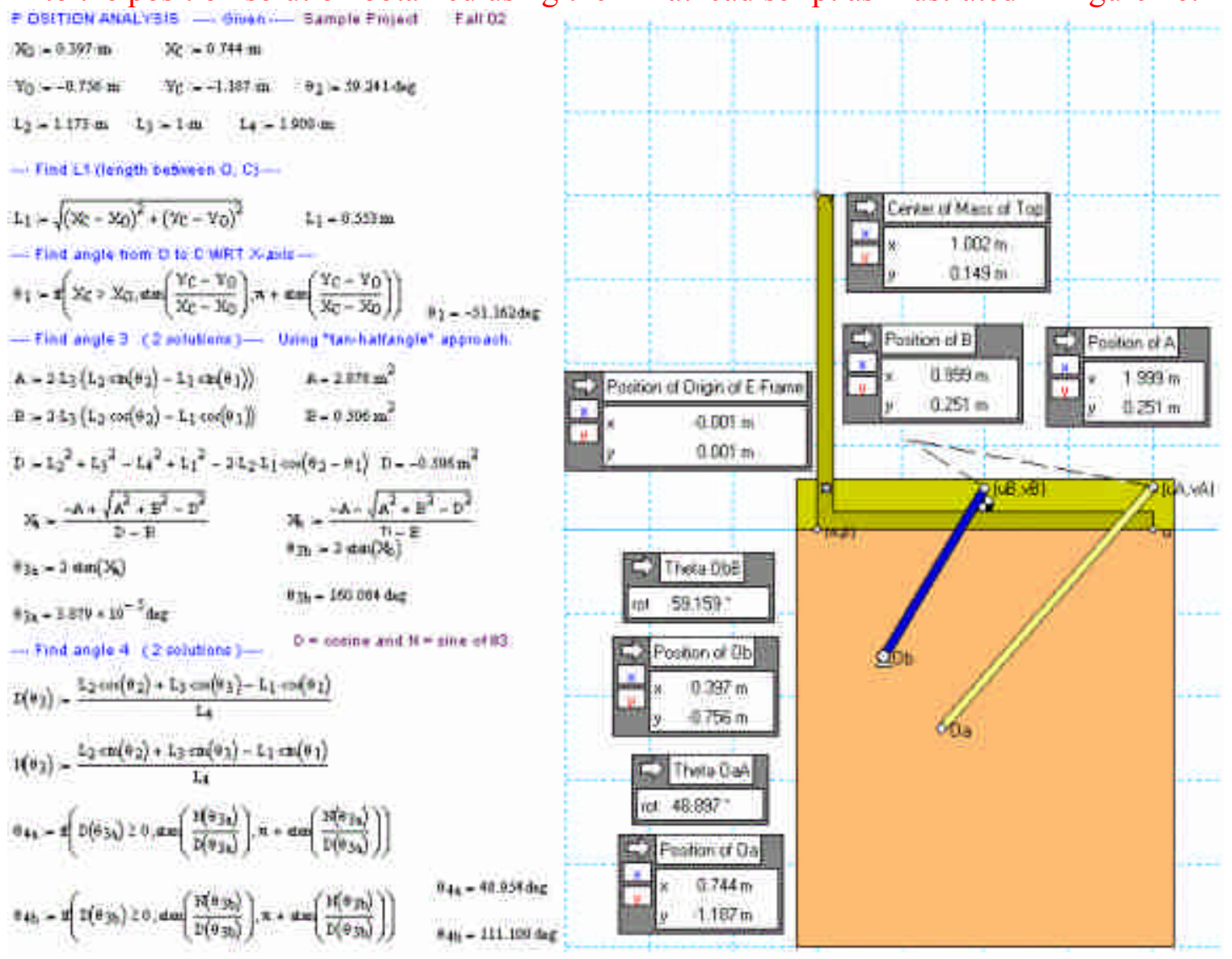

Figure 16. WM2D - Mathcad Position Correlation

2. The students then need to make sure that the link CM's correspond between the WM2D frames and their "Mathcad Frames". In the sample project we need to determine the "Mathcad frame" coordinates of the center-of-mass of the box top. The origin of the Mathcad frame for the top is at point B with the horizontal/u-axis passing through point A. Using the absolute coordinates of the $\mathrm{CM}$ of the top, and points $\mathrm{B}$ and $\mathrm{A}$, the WM2D still frame given above in Figure 16 shows that $\mathrm{u}_{3}=\mathrm{u}_{\mathrm{cm}}=\mathrm{x}_{\mathrm{cmt}}-\mathrm{X}_{\mathrm{B}}=1.000-0.997=0.003$ and $\mathrm{v}_{3}$ $=\mathrm{v}_{\mathrm{cm}}=\mathrm{y}_{\mathrm{cmt}}-\mathrm{y}_{\mathrm{B}}=0.149-0.252=-0.103$. The other two links are thin homogenous bars with their CM's midway between the pivots. 
3. The next step is to set the initial velocities of the links in WM2D. This is accomplished by using their Mathcad script to first solve for the velocities which they then input in the WM2D properties window as illustrated by the Camtasia still frame shown in Figure 17.

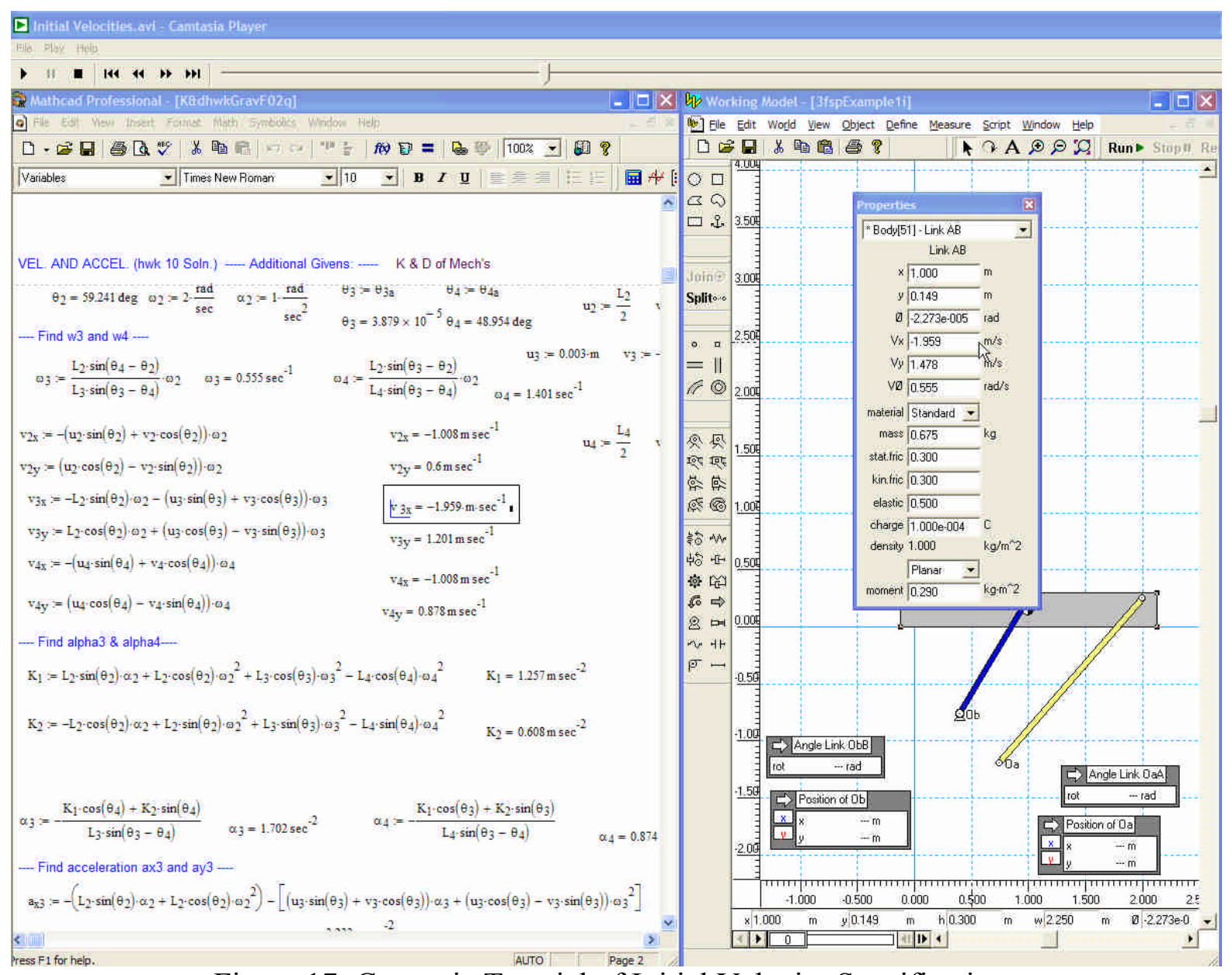

Figure 17. Camtasia Tutorial of Initial Velocity Specification

4. Next the students are shown how to set the motor to an acceleration motor as required to ensure that all aspects of the general solution are verified. By this stage the students need little more than simple graphic direction to complete their WM2D simulation mechanism. 5. They can now "run" a WM2D simulation and verify that the link accelerations obtained by WM2D are the same as those obtained using their Mathcad script.

6. Next the students need to ensure that the masses and inertias correspond. Typically, they use the values obtained in the WM2D properties window for each link and then simply input those into their Mathcad script.

7. Now, they perform the "acid test". They compare the reaction force and required motor torque values obtained from their Mathcad script with the WM2D simulation results as illustrated in Figures 18 and 19.

8. Finally, assuming they have obtained the same results from WM2D and Mathcad, they proceed to dynamically compare their two valid kinematic solutions using WM2D and select one as their "optimal" 4R-4Bar. 


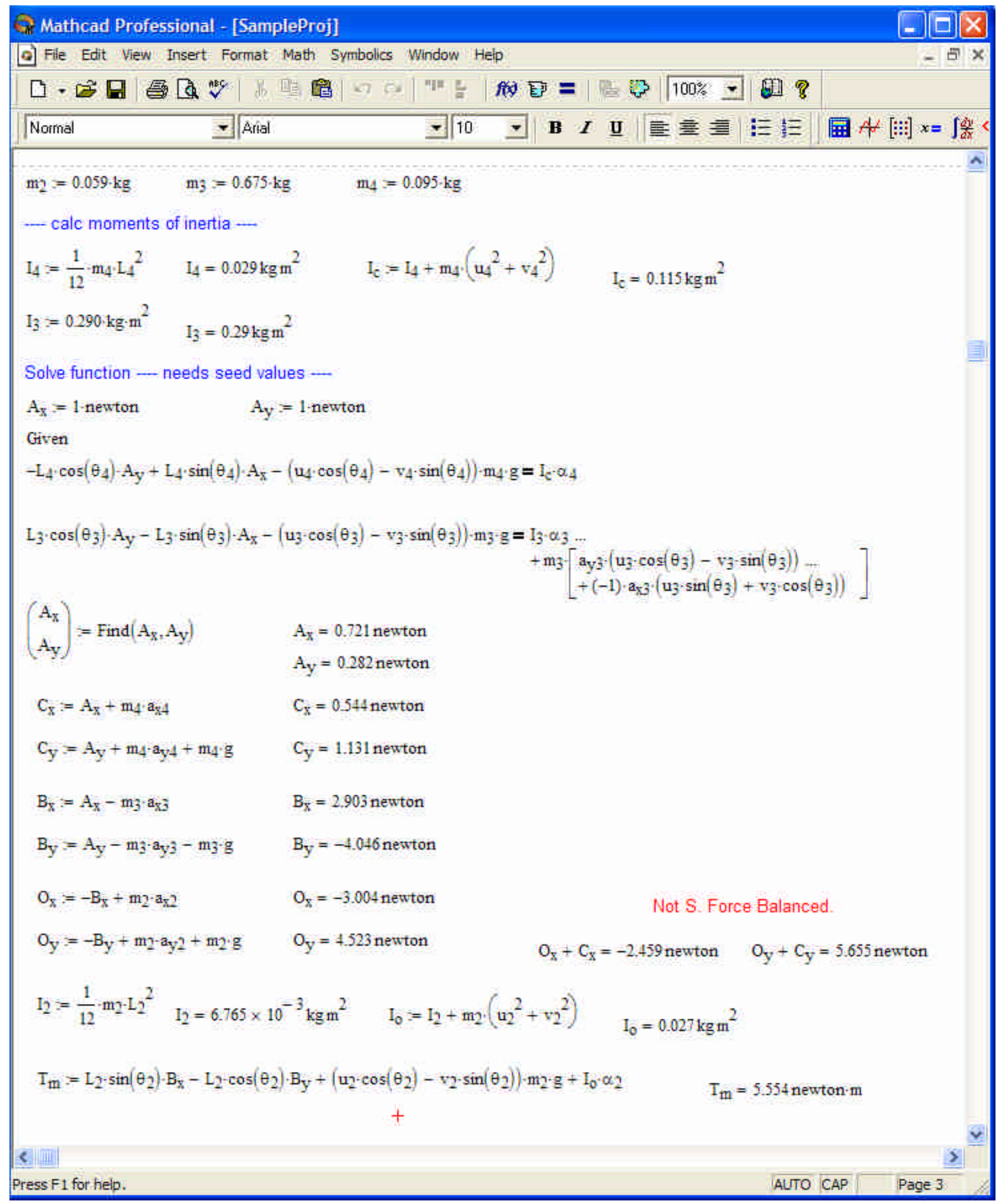

Figure 18. Reaction Forces and Required Motor Torque via Mathcad 


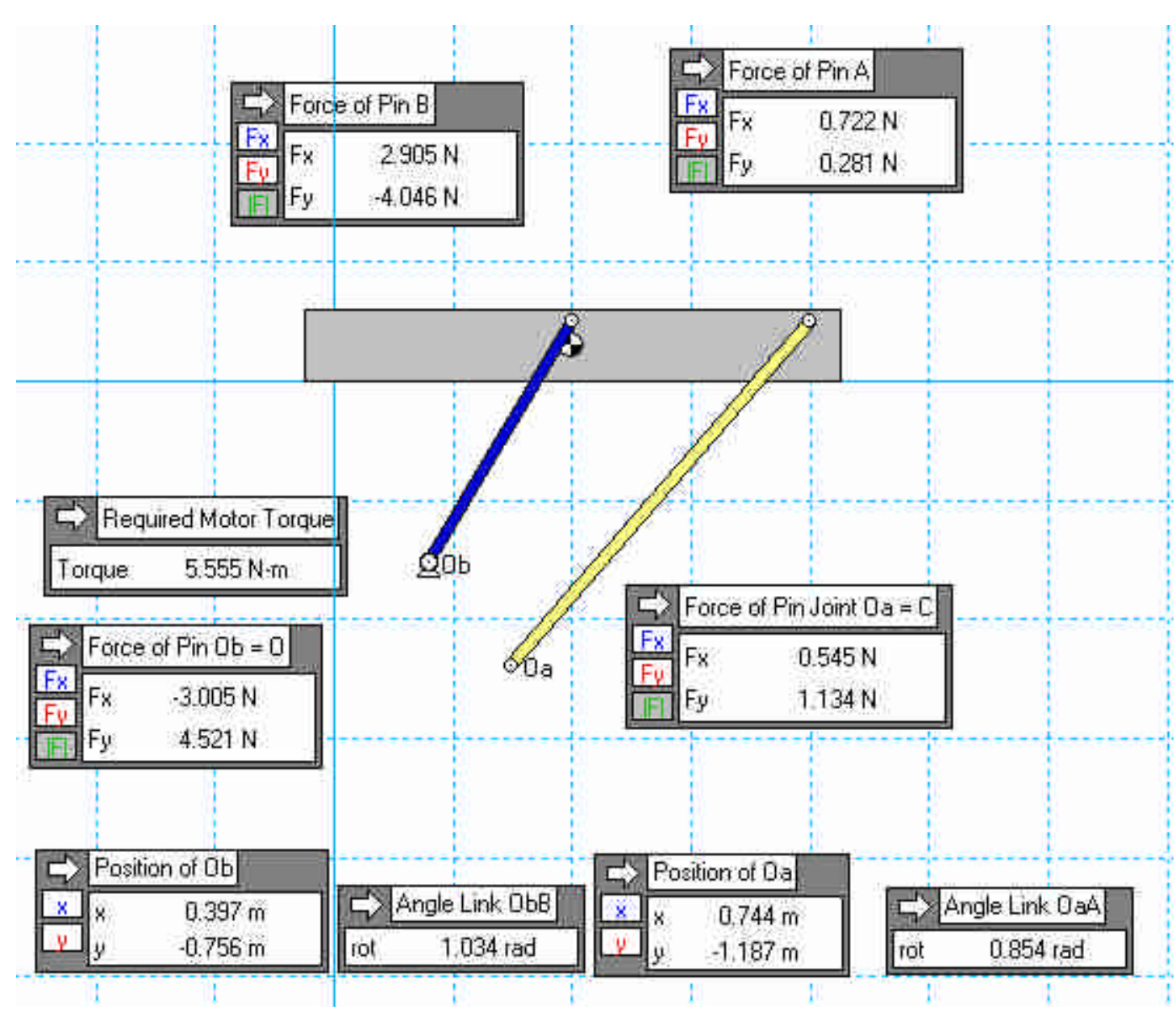

Figure 19. Reaction Forces and Required Motor Torque via WM2D

\section{Conclusion}

The use of "screen capture with audio" avi tutorials, along with a variety of interactive materials including; supplemental lecture notes, homework assignments and solutions, sample exams and exam solutions, and projects has been presented as an approach to asynchronous teaching of math and engineering software, as well as to teaching course content. While there is no hard evidence yet, the results of partial implementation of this approach in Fall 2002 are very promising. The students indicated that they felt that the asynchronous materials not only helped them complete assignments in a timely fashion, but also helped them better understand course concepts. In fact, they were upset when the material development did not keep pace with the class. Full implementation of this approach is planned for Fall 2003, along with formal assessment and evaluation.

Acknowledgments: This work was supported in part by the CCLI-Educational Materials Development Program of the National Science Foundation under Award Number DUE-0089035, the Engineering Research Centers Program of the National Science Foundation under Award Number EEC-9876363, and by The University of Texas System TeleCampus. 